\section{Individualisiertes, modular strukturiertes Patienten- verhaltenstraining bei obstruktiven Atemwegserkrankungen in der stationären Rehabilitation}

\author{
Aktueller Stand des Patientenverhaltenstrainings \\ bei chronischen Atemwegserkrankungen - \\ Erfolge und Grenzen
}

Patientenschulungsmaßnahmen [1] bilden einen zentralen Bestandteil ambulanter wie stationärer medizinischer Rehabilitation. Speziell im Rahmen der pneumologischen Rehabilitationsmedizin ist das Patientenverhaltenstraining als wichtige Komponente inzwischen fest etabliert und Bestandteil der aktuellen nationalen und internationalen Therapieempfehlungen. Dies gilt insbesondere für das Asthma bronchiale [26], aber auch für die COPD [7-9]. Während der letzten 15 Jahre ist eine Reihe von entsprechenden Patientenschulungsbzw. Patientenverhaltenstrainingsprogrammen für Erwachsene wie Kinder entwickelt und evaluiert worden. Zahlreiche empirische Arbeiten zeigen, dass insbesondere beim Asthma bronchiale sowohl verschiedene Verlaufsparameter der $\mathrm{Er}$ krankung wie Notfallaufnahmen, Krankenhaustage, Arbeitsunfähigkeits- und Schulfehltage als auch die Lebensqualität der Patienten günstig zu beeinflussen sind [10-19]. Wie in mehreren nationalen und internationalen Studien nachgewiesen, lassen sich dadurch zudem langfristig deutliche finanzielle Einsparungen erzielen [20,21]. Daher gilt Patientenedukation als essenzieller Bestandteil einer rationalen Therapie chronisch obstruktiver Atemwegserkrankungen. Die Effektivität ist für das Asthma bronchiale gesichert [22], für die chronisch obstruktive Bronchitis als wahrscheinlich anzunehmen $[23,24]$.

\section{Definition, Inhalte und Ziele des Patientenverhaltenstrainings}

Patientenverhaltenstraining bei obstruktiven Atemwegserkrankungen ist die Gesamtheit aller Maßnahmen, die eine aktive Teilnahme des Patienten an der Bewältigung seiner chronischen Krankheit durch Überwachen der Symptomatik und adäquate Selbstanpassung der Therapie an den jeweiligen Schweregrad der Erkrankung ermöglichen [25]. Der Erkrankte soll in die Lage versetzt werden, seine Symptome zu erkennen bzw. zu kontrollieren und Exazerbationen durch rechtzeitige Therapieanpassung $\mathrm{zu}$ beherrschen. Er sollte seine Medikamente mit Wirkungen und Nebenwirkungen kennen und in der Lage sein, diese korrekt anzuwenden (z.B. durch Training der Inhalationstechnik). Ein wichtiges Ziel ist die Akzeptanz der chronischen Atemwegserkrankung, die zusammen mit

Pneumologie 2000; 54: 296-305

(c) Georg Thieme Verlag Stuttgart · New York ISSN 0934-8387
K. Schultz' ${ }^{1}$ M. Schwiersch¹, W. Petro², S. Mühlig ${ }^{3}$,

F. Petermann ${ }^{3}$

${ }^{1}$ Fachklinik Allgäu, Verhaltensmedizinisches Zentrum für Pneumologie, Allergologie, Dermatologie und Psychosomatik, Pfronten-Ried (Chefarzt Dr. K. Schultz)

2 Klinik Bad Reichenhall, Fachklinik für Erkrankungen der Atmungsorgane, Allergologie und für Orthopädie, Bad Reichenhall (Med. Direktor Prof. Dr. W. Petro)

3 Zentrum für Rehabilitationsforschung der Universität Bremen, (Direktor Prof. Dr. F. Petermann)

einem angemessenen, ärztlich begleiteten Selbstmanagement zu einer bestmöglichen Gestaltung von Alltag und Beruf führen soll. Die für diese Zielsetzung erforderlichen Schulungsinhalte (Tab.1) wurden durch die Arbeitsgruppe Patientenschulung der DGP und der Deutschen Atemwegsliga 1995 in einem allgemein akzeptierten Konsensuspapier festgelegt [25].

Ein wesentliches Ergebnis zahlreicher Evaluationsstudien zur Effektivität von „Patientenschulung“ besteht darin, dass Wissensvermittlung alleine keine Verbesserung des Selbstmanagements und der Morbidität bewirkt [26]. Notwendig erscheint vielmehr ein regelrechtes Patientenverhaltenstraining, welches nicht nur eine Verbesserung von Wissen zum Ziel hat, sondern auch das praktische Einüben der erforderlichen Fertigkeiten umfasst und vor allem auf das dauerhafte Etablieren eines krankheitsadäquaten Verhaltens abzielt (Tab. 2). Wissens-, Kompetenz- und Verhaltensänderungen erfordern dabei vielfältige Interventionsmaßnahmen, die sowohl auf kognitiver als auch auf motorischer und emotionaler Ebene greifen. Effektives Patientenverhaltenstraining ist daher weit mehr als bloße Wissensvermittlung!

Gerade in einem stationären Setting bieten sich vielfältige Möglichkeiten für ein systematisches Patientenverhaltenstraining, welches neben „klassischen Schulungskomponenten“ auch spezielle psychologische Bausteine (Krankheitsverarbeitung, Umgang mit der Krankheit im Beruf und im Alltag) einschließt. Zudem bestehen hier ideale Möglichkeiten der systematischen Integration der medizinisch überwachten Sport- und Trainingstherapie in das Patientenverhaltenstraining [27], wobei wesentliche Lerninhalte alltagsnah und praktisch geübt und umgesetzt werden können (z.B. DAGebrauch vor Belastung, Peak-Flow-Kontrolle bei Belastungsdyspnoe, Hustentechnik, Atemtechniken bei Belastung). Die körperliche Trainingstherapie wird somit zu einem praktischen Trainingsfeld des Patientenverhaltenstrainings.

\section{Modulares individualisiertes Patientenverhaltenstraining}

In Deutschland wurde das erste „Asthma-Behandlungs- und Schulungsprogramm“ (ABUS) von der Düsseldorfer Arbeitsgruppe etabliert und evaluiert [28]. Die Erfahrungen dieses stationären Programmes gingen in die ambulanten Schulungsprogramme für Asthma (AFAS = „Ambulantes AsthmaBehandlungs- und Schulungsprogramm“) [29-31] und chronisch obstruktive Bronchitis [32] („Ambulantes Fürther Schulungsprogramm für Patienten mit chronisch obstruktiver Bronchitis und Lungenemphysem“, AFBE) der Fürther Arbeitsgruppe um $\mathrm{H}$. Worth ein. 
Tab. 1 Synopsis der Inhalte des Patientenverhaltenstrainings bei obstruktiven Atemwegserkrankungen nach den Standards der Deutschen Gesellschaft für Pneumologie (DGP) und der Deutschen Atemwegsliga

\section{Unterrichtseinheit}

Namentliche Vorstellung

Einführung in die Problematik

2. Unterrichtseinheit

Aufgaben der Atmung

Kontrolle der Atmung

- Peak-Flow-Meter

- Selbsteinschätzung

3. Unterrichtseinheit

Krankheiten der Atmungsorgane

Einsatz des Peak-Flow-Meters bei Erkrankungen der Atmungsorgane

4. Unterrichtseinheit

Ursachen der Erkrankungen

- Allgemein (hereditär, exogen-allergisch, unspezifisch chemische Noxen, Infekte)

- Speziell (Asthma, chronische Bronchitis, Emphysem)

5. Unterrichtseinheit

Atemwegserkrankungen und Beruf, Familie, Sport, Psyche

6. Unterrichtseinheit

Therapie mit Medikamenten (Früheinsatz, Dauereinsatz)

Medikamentengruppen (Wirkungen, Nebenwirkungen)

Applikationsformen (Vorteile, Nachteile)

7. Unterrichtseinheit

Therapie bei

- Asthma

- Chronische Bronchitis

- Lungenemphysem

Mitbringen der eigenen Medikamente

Peak-Flow-Kurven interpretieren

8. Unterrichtseinheit

Therapie mit Medikamenten

Übungen mit Treibgas-Dosieraerosolen, Pulverinhalatoren, Spacern u.a.

Kontrolle mit dem Peak-Flow-Meter

9. Unterrichtseinheit

Anfall, Exazerbation, Infekt

Atemnotauslösende Mechanismen

Angst vor Asthma

10. Unterrichtseinheit

Therapie mit Kortison (systemische Steroide, topische Steroide, Kortisonangst

11. Unterrichtseinheit

Notfalltherapie

Nicht-medikamentöse Therapie

Atemerleichternde Stellungen

Hustentechniken

12. Unterrichtseinheit

Was wissen wir?

Was leben wir?

Speziell ausgerichtet auf die Bedingungen der stationären Rehabilitation hat das Schulungsteam der Fachklinik Bad Reichenhall unter der Leitung von W. Petro eines der ersten umfassenden Trainingsprogramme für die Gesamtgruppe der chronisch-obstruktiven Atemwegserkrankungen im Erwachsenenalter („Bad Reichenhaller Modell“) entworfen, in die Praxis umgesetzt und in seiner Wirksamkeit dokumentiert [33]. In zwischenzeitlich mehrfach modifizierter Form wurde
Tab. 2 Begriffsdefinitionen: Patienteninformation, Patientenschulung, Patientenverhaltenstraining

- Information: Wissensangebot z. B. mittels Broschüren, Video- oder PC-Programmen.

- Schulung: Systematische Wissensvermittlung nach einem festgelegten Curriculum.

- Patientenverhaltenstraining: Systematische Wissensvermittlung plus motorisch-koordinatives Training (z. B. richtiger Gebrauch von Dosieraerosolen) plus Versuch einer Verhaltensmodifikation durch Beeinflussung sozialer und emotionaler Strukturen (z. B. Lernen in der Gruppe mit Erleben ähnlicher Schicksale und Lösungsstrategien bei anderen. Ein wichtiges praktisches Übungsfeld ist hier die systematische Integration einer speziell für Atemwegskranke konzeptierten körperlichen Trainingstherapie).

dieses Schulungsprogramm von mehreren tausend Patienten erfolgreich absolviert [34].

Trotz vieler Vorzüge dieser ersten stationären Schulungsmodelle, haftete ihnen doch ein wichtiger, konzeptimmanenter Schwachpunkt an. Sie waren bezogen auf den einzelnen Patienten oft zu wenig individuell, und zwar sowohl bezüglich des angebotenen Schulungsumfanges und der Schulungsintensität (z. B. ein einheitlicher Kurs mit festgelegter Stundenzahl für alle Patienten, unabhängig von Aufnahmefähigkeit, Motivation und Schulungsbedarf) als auch insbesondere bezüglich notwendiger spezieller Schulungsinhalte (z.B. gleiche Schulungsintensität des Themas Allergie für allergische und nichtallergische Asthmatiker). Insbesondere im Rahmen der stationären Rehabilitationsmedizin kann es aber nicht darum gehen, den geeigneten Patienten für ein vorhandenes Schulungsprogramm zu finden, sondern das Ziel muss umgekehrt ein flexibles System sein, das an die Bedürfnisse verschiedenster Patientengruppen adaptierbar ist (z.B. spezielle Trainingsmodule für Allergiker oder Sauerstoff-Langzeittherapiepatienten). Konkret besteht die Aufgabe also darin, einen Zwischenweg zu finden zwischen einer unökonomischen und aus Zeitgründen i. d. R. lückenhaften „Individualschulung“ und einer zwar hochgradig standardisierten und strukturierten Gruppenschulung, die aber für einen Teil der Patienten nicht optimal geeignet ist (z.B. für kognitiv weniger strukturierte Patienten oder für Patienten mit Sonderproblemen). Ein möglicher Lösungsweg aus diesem Dilemma besteht in einer konsequent modularen Strukturierung [35] des Patientenverhaltenstrainings: Verschiedene, unabhängig miteinander kombinierbare Schulungs- und Trainingsmodule werden dabei nach dem „Baukastenprinzip“ für jeden Patienten zu einem individuell bedarfsgerechten Curriculum kombiniert. Der Begriff „Modul“ wird hier also nicht im Sinne von „Unterrichtsstunde“ verstanden, sondern beinhaltet jeweils ein thematisch begrenztes, in sich abgeschlossenes Schulungs- oder Trainingsprogramm, welches durchaus mehrere Unterrichtsstunden umfassen kann. Somit kann für jeden Patienten aus einem größeren Pool unabhängiger und in sich geschlossener Schulungs- und Trainingsmodule das für ihn individuell angemessene Curriculum zusammengestellt werden.

Um den Charakter einer Gruppenschulung beizubehalten, ist eine ausreichende Zahl von Patienten mit ähnlichen Schulungsvoraussetzungen erforderlich, um für die einzelnen Schulungsgruppen mit spezifischen Modulangeboten opti- 
male Gruppengrößen von 5 bis 10 (maximal 15) Patienten zu erreichen, mit denen sich einerseits ökonomisch und andererseits effektiv arbeiten lässt. Des Weiteren ist eine genügend große Zahl von qualifizierten Schülern eine Grundvoraussetzung, wobei es sich hier keinesfalls nur um Ärzte handeln muss. Beide Voraussetzungen sind in den größeren pneumologischen Rehakliniken gegeben, so dass diese modulare Schulungsstruktur hier verwirklicht werden kann.

Im Folgenden soll ein modular strukturiertes, individualisiertes Patientenverhaltenstrainingsprogramm am Beispiel der pneumologischen Fachkliniken in Bad Reichenhall und Pfronten dargestellt werden. Das Konzept beruht auf dem Prinzip der individuellen Erstellung eines maßgeschneiderten Schulungscurriculums für jeden einzelnen Patienten, bestehend in der Regel aus einem Intensivtrainingskurs Asthma bzw. Intensivtrainingskurs Chronische Bronchitis/Emphysem, welcher bedarfsweise durch „Essenzialtrainingsmodule“, „Spezialschulungsmodule“ sowie „psychologische Module“ ergänzt wird (s. Tab. 3). Abhängig vom Schulungsbedarf, der Motivation, der Mitarbeitsfähigkeit, den kognitiven Fähigkeiten und der zeitlichen Verfügbarkeit der Patienten ist aber auch eine Zusammensetzung des individuellen Schulungprogrammes nur aus „Essenzialtrainingsmodulen“ (Konzept der essenziellen Minimalschulung) und/ oder „Spezialtrainingsmodulen“ sinnvoll und möglich.

Tab. 3 Differenzierungsaspekte des modular strukturierten Patientenverhaltenstrainings

- Intensivkurse: Separate Wochenkurse „Asthma bronchiale“ bzw. "Chronische Bronchitis/Emphysem“

- Essenzialtrainingsmodule: Einüben essenzieller praktischer Fertigkeiten

- Spezialtrainingsmodule (unterschiedliche individuell notwendige Schulungsthemen)

- Psychologische Module

1. Intensivkurse „Asthma bronchiale“ bzw. „Chronische Bronchitis/ Emphysem":

Separate einwöchige Intensivkurse zur Vermittlung der relevanten Kenntnisse und Fertigkeiten für ein krankheitsadäquates Verhalten

- Intensiv-Wochenkurs: Asthma bronchiale

- Intensiv-Wochenkurs: Chronische Bronchitis/Emphysem

2. Essenzialtrainingsmodule:

- Modul: Inhalative Medikamente bei Asthma und Bronchitis

- Modul: Krankheitsselbstkontrolle mittels Peak-Flow-Meter

3. Spezialtrainingsmodule:

- Allergikertrainingsprogramm (pro Woche je 2 Module à 1 Doppelstunde in einem 14-tägigen Turnus)

- Grundlagenmodul

- Modul für Hausstaubmilbenallergiker

- Modul für Pollenallergiker

- Modul für Tier- und Schimmelpilzallergiker

- Modul: Therapie mit elektrischen Inhalierapparaten (pro Woche 1 Kurs à 1 Doppelstunde)

- Modul: Sauerstoff-Langzeittherapie (2 Doppelstunden alle 14 Tage)

- Modul: Schlafapnoe (1 Doppelstunde)

4. Psychologische Module

- Modul: Interozeptionstraining

- Modul: Selbstsicherheitstraining

- Modul: Selbstmanagement

- Modul: Nichtraucher werden
Derzeit erfolgt die Indikationsstellung für die verschiedenen Module vor allem durch die behandelnden Stationsärzte. Bei eindeutigen Diagnosen ist dies für viele Patienten unproblematisch (z.B. nichtgeschulter, motivierter Emphysempatient mit Indikation zur Sauerstoff-Langzeittherapie $\rightarrow$ mögliches Curriculum: Intensivtrainingskurs „Chronische Bronchitis/ Emphysem“ + Spezialmodul „Sauerstoff-Langzeittherapie“ + Spezialmodul „Apparateinhalation“ + COPD-Sportgruppe). Darüber hinaus geht in die ärztliche Indikationsstellung aber auch immer eine intuitive Einschätzung des Schulungsbedarfes, der Motivation und Mitarbeitsfähigkeit des Patienten mit ein. Idealerweise sollte dieser Teil der Indikationsstellung aber auf einer systematischen Patiententypologie basieren, d.h. es wäre für die Zukunft wünschenswert, die Zuweisungskriterien expliziter zu definieren und soweit wie möglich zu standardisieren. Voraussetzung solches Patienten-Screenings wäre eine Art vorgeschalteter Schulungsdiagnostik, die zumindest drei zentrale Patientenmerkmale zu identifizieren hätte:

- individueller Schulungsbedarf: Vorwissen, Selbstmanagement-Kompetenzen, Belastungsgrad, Copingfähigkeiten, spezifische Sonderprobleme;

- individuelle Schulbarkeit: Alter, kognitive Funktionsfähigkeit (Verständnis, Erinnerungsfähigkeit, Sprache), Krankheitseinstellungen (Akzeptanz, Kontrollüberzeugung, Selbstwirksamkeit);

- individuelle Schulungsbereitschaft: Interesse, Motivation, persönliche Sichtweisen und Ziele.

Es erscheint uns wahrscheinlich, dass dadurch das „Konzept des individualisierten, modular strukturierten Patientenverhaltenstrainings" noch zu optimieren wäre. Hier besteht aber noch ein erheblicher Forschungs- und Entwicklungsbedarf (valide und reliable Diagnostika).

Durch den obligaten Gebrauch von Overhead-Folien, die die wesentlichen Lehrinhalte in Form von stichwortartigen Texten und Merksätzen enthalten, wird eine sehr weitgehende Standardisierung der Schulungsinhalte und -methoden erreicht. Dadurch ist es möglich, ein solch komplexes System von Krankheits- und Behandlungsinformationen durch verschiedene bzw. wechselnde Schuler zu vermitteln, ohne dass die Übertragbarkeit der Ergebnisse gefährdet wird. Durch die Standardisierung wird zudem gewährleistet, dass die Inhalte und die Durchführung unabhängig von der schulenden Person konstant bleiben. Dies ist die Voraussetzung, dass ein Schulungs-/Verhaltenstrainingsprogramm auch mit wechselnden Schulern kontinuierlich über Jahre in jedem Rehadurchgang in vergleichbarer Weise (nach demselben Curriculum und Stundenplan) durchführbar ist. Zudem wird der durch den Folieneinsatz erreichbare Zeitgewinn konsequent für das praktische Einüben der erforderlichen Grundfertigkeiten mit allen Schulungsteilnehmern - unter Supervision durch die Gruppe - genutzt. Dies betrifft vor allem das systematische Einüben der korrekten Dosier-Aerosol-Technik, die Ermittlung der „Peak-Flow-Ampelzonen“ und das praktische Durchspielen möglicher Notfallsituationen. 
Tab. 4 Curricula der Intensivkurse (Grundlagenmodule) „Asthma bronchiale“ und „chronische Bronchitis/Emphysem“ der Fachkliniken in Bad Reichenhall und Pfronten

A) Curriculum des Intensivkurses „Asthma bronchiale“ der Fachkliniken in Reichenhall (8 Stunden) u. Pfronten (10 Stunden)

\begin{tabular}{|c|c|c|c|c|c|}
\hline & Montag & Dienstag & Mittwoch & Donnerstag & Freitag (Pfronten) \\
\hline \multirow[t]{2}{*}{60 Minuten } & 1. Stunde & 3. Stunde & 5. Stunde & 7.-8. Stunde & 9.-10. Stunde \\
\hline & $\begin{array}{l}\text { Vorstellung } \\
\text { Was wollen wir } \\
\text { erreichen? } \\
\text { Wie funktioniert } \\
\text { Patientenschulung? }\end{array}$ & $\begin{array}{l}\text { Medikamentenkunde } \\
\text { Stufentherapie des } \\
\text { Asthma bronchiale }\end{array}$ & $\begin{array}{l}\text { Selbstkontrolle } \\
\text { (Symptome, DA-Bedarf, } \\
\text { Peak-Flow-Meter) } \\
\text { Akuter Bronchialinfekt } \\
\text { (Vorbeugung, Erken- } \\
\text { nung, Bedeutung und } \\
\text { Therapie) }\end{array}$ & $\begin{array}{l}\text { Psychologische } \\
\text { Aspekte: Phasen der } \\
\text { Krankheitsverarbeitung, } \\
\text { soziale Aspekte der } \\
\text { Krankheit. } \\
\text { Krankheitseinstellung } \\
\text { und Selbstmanagement }\end{array}$ & $\begin{array}{l}\text { Wiederholungsstunde: } \\
\text { (Schwerpunkt: Meine } \\
\text { Medikamente, mein } \\
\text { Selbstmanagement, } \\
\text { meine Notfalltherapie) }\end{array}$ \\
\hline \multirow[t]{2}{*}{60 Minuten } & 2. Stunde & 4. Stunde & 6. Stunde & & \\
\hline & $\begin{array}{l}\text { Aufbau und Funktion } \\
\text { der Atmungsorgane, } \\
\text { Asthma als chronische } \\
\text { Entzündung der Bron- } \\
\text { chialschleimhaut mit } \\
\text { bronchialer Hyper- } \\
\text { reagibilität } \\
\text { Krankheitslehre: } \\
\text { Allergisches, nicht- } \\
\text { allergisches, gemischt- } \\
\text { förmiges Asthma Son- } \\
\text { derformen (Anstren- } \\
\text { gungsasthma, „Medika- } \\
\text { mentenasthma“) }\end{array}$ & $\begin{array}{l}\text { Nicht-medikamentöse } \\
\text { Therapie des Asthmas }\end{array}$ & $\begin{array}{l}\text { Selbstmanagement } \\
\text { Ampelschema } \\
\text { Notfalltherapie }\end{array}$ & & \\
\hline
\end{tabular}

B) Curriculum des Intensivkurses „chronische Bronchitis/Emphysem der Fachkliniken in Reichenhall (8 Stunden) und Pfronten (10 Stunden). Beide Grundlagenmodule finden z. B. in Pfronten jede Woche parallel statt

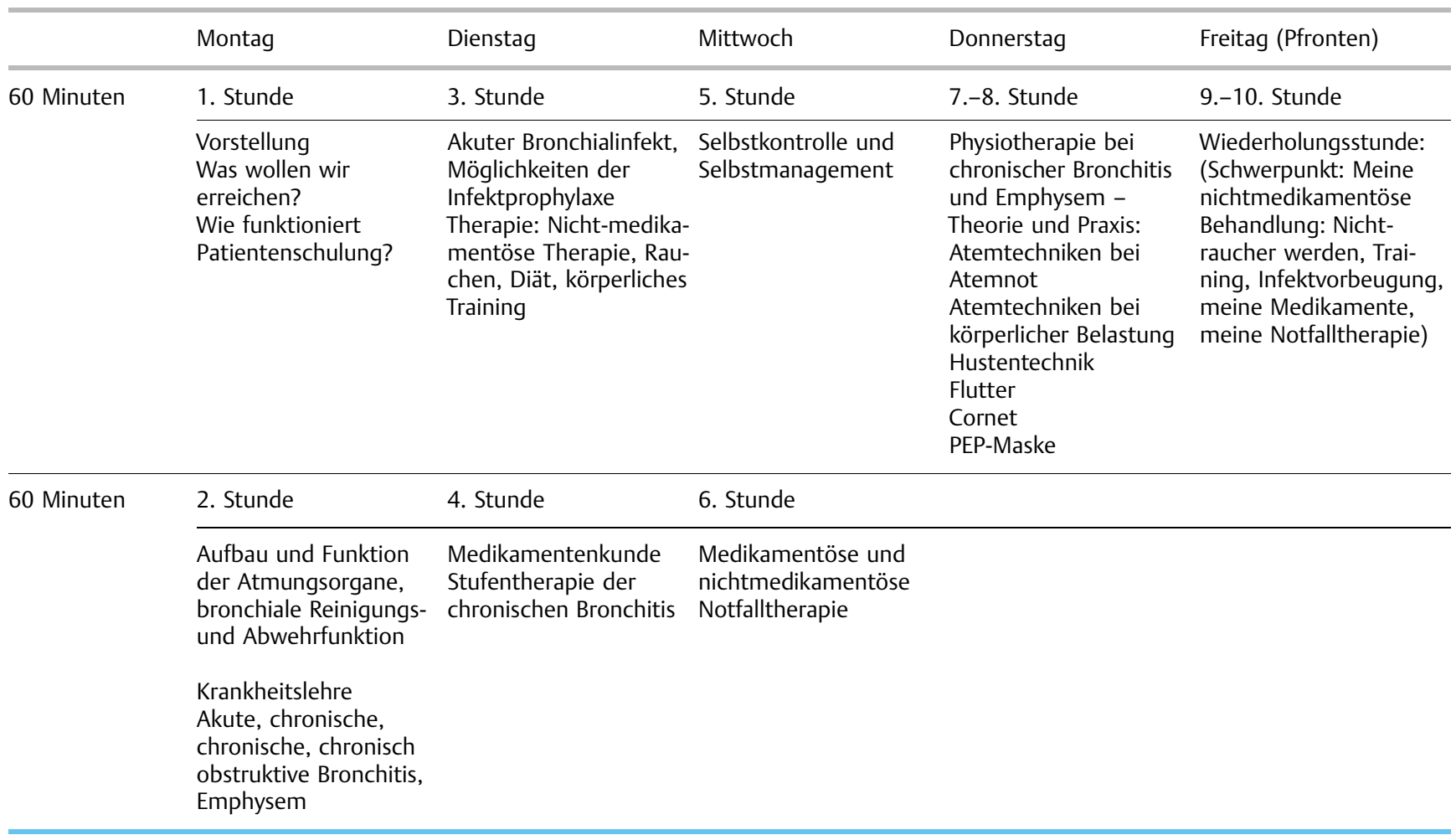


Intensivkurs „Asthma bronchiale“, Intensivkurs „chronische Bronchitis/Emphysem“

Im Rahmen dieser einwöchigen Intensivkurse werden in Gruppen mit zumeist 8-12 Teilnehmer das erforderliche Wissen und die nötigen praktischen Fertigkeiten, entsprechend den o.g. Empfehlungen (Tab.1), vermittelt und eingeübt. Diese Wochenkurse beinhalten neben Stunden mit vorwiegender „Wissens- und Fertigkeitsvermittlung“ („Aufbau der Atmungsorgane“, „Krankheitslehre“, „,medikamentöse und nicht-medikamentöse Therapie“, „Selbstkontrolle“) auch Unterrichtseinheiten mit primär psychologischen Themen sowie thematisch offene „Zusammenfassungsstunden“. Die Durchführung erfolgt interdisziplinär durch fachlich entsprechend weitergebildete Ärzte, Sozialpädagogen und Psychologen. Die Indikationsstellung wird durch die Stationsärzte vorgenommen und verlangt spezielles pneumologisches Wissen, aufgrund der keineswegs immer leichten Zuordnung der Patienten zu einer der beiden Gruppen.

Medizinische Inhalte der Intensivkurse „Asthma bronchiale“ und „Chronische Bronchitis/Emphysem“

Die „medizinisch-ärztlichen Inhalte“ dieser einwöchigen Schulungskurse sind in Tab. 4 aufgelistet. Angelehnt an die oben erwähnten Standards (Tab.1) sind die dort geforderten Inhalte für den Asthma- bzw. Bronchitis/Emphysemkurs jeweils inhaltlich differenziert vorgegeben. So wird im COPDKurs beispielsweise besonderes Gewicht auf die Themen Rauchen, Atem- und Hustentechniken, Schleimmobilisation und körperliches Training gelegt. Eindeutige Schwerpunkte des Asthmakurses sind die medikamentöse Therapie, die Selbstkontrolle, das Peak-Flow-Meter-gesteuerte Selbstmanagement sowie das Verhalten beim schweren Asthma-Anfall. Selbstverständlich gibt es aber in beiden Kursen auch viele thematische Gemeinsamkeiten, wie den Curricula zu entnehmen ist (Tab. 4).

Psychologische Inhalte der Wochenkurse „Asthma bronchiale“ und "Chronische Bronchitis/Emphysem“

Die „Einführungs- und Vorstellungsstunde“ (1. Stunde) dient dem gegenseitigen Kennenlernen von Patienten und Trainern, der Vorstellung der Methodik, der inhaltlichen Einführung in das Verhaltenstraining und der Motivation der Patienten als Basis für eine aktive Mitarbeit. Individuelle Voraussetzungen, Bedürfnisse und Erwartungen der Patienten sind abzuklären. Nach der Schaffung einer förderlichen Beziehung können Unterschiede in Krankheits- und Behandlungskonzepten zwischen Behandlern und Patienten thematisiert werden [37], um eine ausreichende Kongruenz zu erreichen. Die Doppelstunde „Leben mit der Erkrankung“ (7./8. Stunde) dient der Bahnung einer förderlichen Einstellung gegenüber der eigenen Erkrankung, der Veränderung problemstabilisierender Kognitionen („gesund“ - „krank“) sowie der Förderung eines selbstverständlichen Umgangs mit der Erkrankung.

\section{Essenzialtrainingsmodule - Konzept der essenziellen Minimalschulung}

In Deutschland existieren inzwischen zahlreiche, z.T. auch evaluierte Schulungsmodelle mit einem definierten Curriculum, sowohl für die Gesamtgruppe der obstruktiven Atem- wegserkrankungen als auch differenziert für Asthma und Bronchitis/Emphysem (Übersicht bei [38] und [39]). Beispiele sind

- das Modell des Berufsverbandes der Pneumologen,

- die ambulanten Fürther Schulungsmodelle für Asthma bzw. chronische Bronchitis/Emphysem

- sowie verschiedene von der Industrie verbreitete Modelle.

Dabei wird bei den Curricula dieser Programme nicht systematisch nach Lernzielumfang und kognitivem Niveau für verschiedene Gruppen differenziert. Überspitzt mag dies im Einzelfall auf die Entscheidung „schulbar bzw. nicht schulbar“ hinauslaufen. Maßgeschneiderte, individualisierte Schulungsprogramme, gerade im Rahmen der stationären Rehabilitation, müssen aber auch Optionen für Patienten bieten, die mit einer 6-10-stündigen Gruppenschulung (komplettes Curriculum entsprechend den DGP-Richtlinien) überfordert sind. Hier bietet sich konsequenterweise die Beschränkung auf eine essenzielle Minimalschulung an, also die Konzentration auf die Vermittlung essenzieller Mindestfertigkeiten und -kenntnisse (1-2 Stunden). Dies ist mit den im Folgenden dargestellten „Essenzialtrainingsmodulen“ intendiert. Die Erarbeitung dieser Essenzialtrainingsmodule war daher ein entscheidender Schritt zur Verwirklichung des modularen Schulungskonzeptes. Wir verstehen darunter separate Trainingsbausteine, die sich auf das gezielte Einüben und Trainieren zentraler und unabdingbarer („essenzieller“) Grundfertigkeiten und Kenntnisse beschränken, die für das Selbstmanagement chronisch obstruktiver Atemwegserkrankungen unerlässlich sind. Dazu gehören in erster Linie der richtige Gebrauch der verschiedenen Dosieraerosol-/Pulverinhalations-Systeme (Essenzialtrainingsmodul: „Inhalative Medikamente bei Asthma und Bronchitis") sowie die Selbstüberwachung mittels Peak-Flow-Meter-Kontrolle (Essenzialtrainingsmodul: „Peak-Flow-Meter“ [40]). Diese Essenzialtrainingsmodule, die mit allen anderen Kursen bzw. Modulen frei kombinierbar sind, haben zwei z.T. unterschiedliche Funktionen:

- Wiederholung und Vertiefung: Zeigen sich bei der routinemäßigen Kenntnis- und Fertigkeitsüberprüfung (z.B. im Rahmen der Visite nach den „allgemeinen Trainingskursen“ und der individuellen Einführung durch Arzt und/oder Schwester) noch Lücken oder Unklarheiten bei der Beherrschung dieser Essenzialfertigkeiten, so erfolgt in diesen Kursen eine nochmalige und gezielte Instruktion und Übung.

- Separatangebot der essenziellen Minimalschulung: Für manchen Patienten ist bspw. ein vollständiges Asthmatikerschulungsprogramm zu umfangreich, sei es aus Gründen der Motivation oder der kognitiven Kapazität. In diesen Fällen kann aber ein speziell auf die individuellen Bedürfnisse zugeschnittenes, gezieltes Minimalprogramm indiziert und nützlich sein (z.B. bestehend nur aus dem Essenzialtrainingsmodul zum korrekten Gebrauch der Dosieraerosole). Die Essenzialtrainingsmodule sind also so konzipiert, dass sie auch separat und unabhängig von anderen Schulungsinhalten als Minimal-Schulungsprogramm fungieren können.

Das Konzept der essenziellen Minimalschulung, also der konsequenten Beschränkung auf ein aus modularen Komponenten zusammengestelltes Schulungscurriculum für die individuell erforderlichen Mindestfertigkeiten und -kenntnisse 
wäre auch an Akutkrankenhäusern oder im Rahmen einer ambulanten Patientenschulung umsetzbar, wo die vorhandenen Ressourcen an Zeit und Schulungspersonal zu einer Beschränkung auf die essenziellen Inhalte zwingen. Aufgrund der hochgradigen Standardisierung dieser Essenzialtrainingsmodule ist der Lerneffekt seitens der Patienten auch bei Schulern unterschiedlicher Professionen vergleichbar ausgeprägt. Dies wurde exemplarisch am Modul des Peak-FlowMeter-Trainings demonstriert [41]. Daher können diese Essenzialtrainingsmodule genauso effektiv durch nichtärztliche Trainer durchgeführt werden [42], was in Bad Reichenhall und Pfronten erfolgreich praktiziert wird. In einer prospektiven und randomisierten Studie erwies sich dieses standardisierte und strukturierte Vorgehen in seiner Effizienz den weniger exakt definierten Schulungsformen sowohl bezüglich der Wissensvermittlung (Multiple Choice-Fragen) als auch bezüglich der erlernten praktischen Fertigkeiten (Beobachtungsscore) und des tatsächlichen durchgeführten Verhaltens (Patiententagebuch) als überlegen. Zudem zeigte sich gegenüber dem alleinigen Intensivwochenkurs („Asthma bronchiale“) ein zusätzlicher Benefit [43].

Computerbasierte Schulungsmodule: Aufgrund der hochgradigen Standardisierung war die Umsetzung der „Wissensinhalte" der Essenzialtrainingsmodule in Form von PC-basierten Schulungsprogrammen naheliegend. Zu betonen ist, dass solche PC-Programme zwar das konventionelle Patientenverhaltenstraining effektiv ergänzen, aber keinesfalls ersetzen können und sollen. Letztlich sind hierdurch vor allem Wissensinhalte, weniger aber die praktischen Fertigkeiten und psychosozialen Aspekte der Krankheitsbewältigung trainierbar. Ziel des ergänzenden und fakultativen Einsatzes innerhalb eines stationären Schulungssystems ist, die damit zu gewinnenden personellen und zeitlichen Einsparungen dem eigentlichen umfassenden Patientenverhaltenstraining zugute kommen zu lassen. BREATH (Bad Reichenhaller elektronische Asthma-Therapie-Hilfe) ist ein an der Klinik Bad Reichenhall entwickeltes interaktives und multimedial gestaltetes PCPatientenschulungs-Programm [44,45]. Es basiert auf dem evaluierten Essenzialtrainingsmodul („Peak-Flow-Meter-Training“), geht über dieses jedoch weit hinaus und umfasst die wesentlichen Aspekte des gesamten Selbstmanagementtrainings bei Asthma bronchiale, vor allem Krankheitsverlaufskontrolle und medikamentöse Therapie. Zwar liegt der Schwerpunkt auf einer detaillierten Einführung in die Selbstkontrolle mittels Peak-Flowmetrie, es werden aber ebenso detailliert die Prinzipien der Stufentherapie erläutert und in zahlreichen praxisnahen Trainingssequenzen interaktiv eingeübt. Das Programm hat reges Interesse gefunden und eine erste Pilot-Evaluation wurde erfolgreich abgeschlossen. Hierbei konnte neben einem deutlichen Wissenszuwachs vor allem die Akzeptanz des Mediums PC seitens der Patienten dokumentiert werden [46]. Zwischenzeitlich ist das zweite BREATH-Modul fertiggestellt: „More BREATH" beinhaltet die Umsetzung des Essenzialtrainingsmoduls „Inhalative Medikamente“; weitere Module („Allergo-BREATH“ = Umsetzung der verschiedenen evaluierten Allergiker-Trainingsmodule, „Oxygen-BREATH“ = Umsetzung des Spezialmoduls zur $\mathrm{O}_{2}$-Langzeittherapie) werden derzeit erstellt bzw. sind in Vorbereitung.

\section{Spezialtrainingsmodule [47]}

Ein einheitliches Gesamt-Curriculum „Asthma, Bronchitis, Emphysem“ muss zwangsläufig bei vielen Patienten mit „Sonderproblemen“ unvollständig bleiben. Diese sind jedoch gerade in einer pneumologischen Schwerpunkt-Rehabilitationsklinik überproportional vertreten. Es liegt auf der Hand, dass ein weiterer spezieller Schulungs- und Trainingsbedarf bei verschiedenen Patientenuntergruppen besteht, der durch ein konventionelles Schulungsprogramm nicht ausreichend abgedeckt wird. Für diese Patienten sind spezielle Schulungsund Trainingsprogramme vonnöten. Dies betrifft z.B. Patienten mit:

- Allergien,

- Indikation zur Sauerstoff-Langzeittherapie,

- Indikation zur intermittierenden Selbstbeatmung (ISB),

- Schlafapnoe (nCPAP, BiPAP)

- Inhalationsgeräten (Düsenvernebler, IPPB)

- interstitiellen Lungenerkrankungen

- Bronchiektasenerkrankung

- fremdsprachige Patientenschulung usw.

Spezialtrainingsmodule sehen wir als optionale Zusatzbausteine (Module) eines modular strukturierten Gesamtprogrammes. Ziel ist letztlich eine umfassende Palette frei kombinierbarer Schulungs- und Trainingsmodule, so dass für jeden Patienten ein auf seine individuellen Bedürfnisse maßgeschneidertes Patientenverhaltenstraining angeboten werden kann.

Beispiel 1: Modular strukturierte AllergikerKarenztrainingsprogramme [48]

Als Teil des für jeden Einzelfall individuell erstellten Curriculums durchlaufen jene Patienten mit einer klinisch relevanten Atemwegsallergie ein „allergenspezifisches Allergikertraining“, welches aus einem Grundlagenmodul und drei optionalen Trainingsgruppen (Hausstaubmilben [49], Pollen, Tiere/ Schimmelpilze) besteht. Für jeden Patienten kann so ein individuelles Allergikertrainingsprogramm zusammengestellt werden. Zudem ist es dadurch möglich, das allgemeine Schulungsprogramm von diesen Sonderthemen weitgehend zu entlasten. Das „Allergikertraining“ erfolgt in Kleingruppen und findet in einem zweiwöchentlichen Turnus statt. Jedes Modul umfasst eine Doppelstunde. Die Indikationsstellung erfolgt durch die behandelnden Ärzte, Einschlusskriterium ist eine gesicherte und klinisch relevante Sensibilisierung gegen die genannten Allergene. Im Grundlagenteil („Grundlagenwissen für Atemwegsallergiker“) werden Fragen besprochen wie: „Was ist Allergie, wie entsteht sie, wie wird sie diagnostiziert?" Des Weiteren werden die Grundprinzipien der Therapie behandelt sowie die Themenkomplexe „Vererbung Vorbeugung - Haustiere - Berufswahl“. Dieses „AllergieGrundlagenmodul" ist für alle Patienten identisch. Anschließend erfolgt die gezielte Vermittlung weiterer spezifischer Kenntnisse und Kompetenzen in drei speziellen „Aufbaumodulen". Hier wird besonderer Wert auf ein praktisches Einüben der notwendigen Kenntnisse und Fertigkeiten gelegt, unter besonderer Betonung der Karenzmaßnahmen. Das Programm besteht einerseits aus weitgehend ausformulierten Texten (Overhead-Folien, Kurshefte) und Dias/Videofilmen (multimedial), andererseits aus praktischen Übungen und Demonstrationen. Die vorliegenden Evaluationsergebnisse 
[50] bestätigen die Effektivität dieses Allergikertrainings, gemessen am Grad der Wissensvermittlung und der Akzeptanz seitens der Patienten.

\section{Beispiel 2: Trainingsprogramm Sauerstoff-Langzeittherapie}

Die Einleitung einer solchen Therapie stellt für den Patienten eine einschneidende Veränderung seiner gesamten Lebenssituation dar. Die Effektivität dieser Behandlungsform hängt ganz entscheidend von der konsequenten Therapiedurchführung ab. Eine solche verlangt aber nicht nur ein erhebliches Maß an Motivation seitens des Patienten, sondern auch eine sehr intensive und gründliche Einweisung durch den Arzt. Grundlage einer tragfähigen Motivation ist das Wissen um die Zusammenhänge und die Prognose des Cor pulmonale und die Möglichkeit, letztere durch eine Sauerstoff-Langzeittherapie entscheidend zu verbessern. Wesentlich erscheint insbesondere die Vermittlung der Tatsache, dass eine solche Behandlung nicht primär auf die Besserung von Husten, Auswurf und Dyspnoe zielt. So wird Sauerstofftherapie sehr oft mit „besser Luft bekommen“ gleichgesetzt. Auf solchen Missverständnissen beruht sicher ein Teil der fehlenden Compliance. Neben der Vermittlung dieser notwendigen Grundkenntnisse muss aber vor allem die praktische Durchführung der Therapie eingeübt werden. Oft erst während dieser Übungsphase ergeben sich die konkreten Fragen seitens der Patienten. Da sich all diese Lernanforderungen gut im Rahmen eines speziellen stationären Trainingsprogrammes durchführen lassen, wurde 1995 an der Klinik Bad Reichenhall ein standardisiertes Trainingsprogramm zur $\mathrm{O}_{2}$-LZT entwickelt [51], welches von entsprechenden Patienten seither routinemäßig durchlaufen wird. Ziel des Kurses ist sowohl Wissensvermittlung über den Zweck und notwendigen Umfang der $\mathrm{O}_{2}$-LZT, als auch ein praktisches Training vieler technischer Details (Lärmvermeidung, Mobilität, Hygiene, Filterwechsel, Fragen bez. Reisen u. Berufstätigkeit u.a.). Auch dieser Kurs ist in einen einleitenden theoretischen Grundteil und einen eher praktischen Unterweisungsteil gegliedert. Entscheidend scheint vor allem das Demonstrieren und selbstständige Ausprobieren der verschiedenen Sauerstoffsysteme sowie der verschiedenen Nasensonden. Dieses praktische Einüben ist insbesondere bei mobilen Systemen zwingend erforderlich. Wesentlich ist hier auch der emotionale Benefit der Patienten (z.B. Erleben und gemeinsames Angehen eines ähnlichen Lebensschicksals). Auch für dieses Spezialmodul bestätigen die vorliegenden Evaluationsergebnisse die Effektivität gemessen am Grad der Wissensvermittlung, der Verbesserung der praktischen Fertigkeiten und der Akzeptanz seitens der Patienten [52,53].

Verschiedene internationale Arbeiten untersuchten die Compliance bei der Durchführung der LTOT und zeigten erhebliche Mängel bei der praktischen Durchführung [54,55]. So wurde die Therapie bei etwa der Hälfte der untersuchten Personen nicht korrekt durchgeführt [56]. In der Literatur finden sich allenfalls sporadisch Arbeiten zur Frage der Auswirkung von Patientenschulung auf die Compliance und Effektivität einer Sauerstoff-Langzeittherapie. Insbesondere zur Prüfung der Effektivität einer solchen Schulung auf die Dauer der täglichen $\mathrm{O}_{2}$-Insufflation existieren nur zwei Studien, wobei sich immerhin Hinweise auf einer Verbesserung der täglichen Anwendungszeit durch Schulung fanden
$[57,58]$. Kontrollierte prospektive Studien zu dieser Fragestellung fehlen aber bislang.

\section{Ergänzende psychosoziale Module}

Ein verhaltensmedizinisch optimiertes Schulungsmodell verlangt die Integration psychologischer Verfahren zur Verbesserung des Umgangs mit chronischer Erkrankung und zur Verhaltensmodifikation. Neben begleitenden therapeutischen Elementen wie Entspannungstraining, Sport- und Ernährungstherapie sollen die bereits bestehenden Module einerseits - wie oben beschrieben - psychologisch optimiert und andererseits durch neue Modulelemente ergänzt werden. Besonderes Augenmerk wird dabei auf die Krankheitsbewältigung, die Verbesserung der Motivation, der Selbstmanagementkompetenzen, der Interozeptionsfähigkeit und auf einen selbstsicheren Umgang mit sozialen Aspekten der Erkrankung gelegt, um damit die Compliance langfristig zu unterstützen [59].

\section{Modul Interozeptionstraining}

Wie die psychophysiologische Grundlagenforschung belegt, lässt sich durch gezieltes Interozeptionstraining die Symptomwahrnehmung von Asthmatikern deutlich verbessern [60 - 63]: Das systematisches Wahrnehmungstraining für körperinterne Prozesse zielt darauf $a b$, eine geräteunabhängige Sensibilisierung für Frühwarnzeichen einer Verschlechterung zu vermitteln (Atemwegsobstruktionen, Symptomfrüherkennung), individuelle Warnsignale und Auslösermuster identifizieren zu lernen sowie die Effekte der Medikation sinnlich erfahrbar zu machen. Während des Kurses wird die Bedeutung der Interozeption erarbeitet, die die Eigenkontrolle mittels Peak-Flow-Meter unterstützen soll. Die Patienten erhalten einen Protokollbogen, auf dem sie in der Art eines „Körperchecks“ relevante Symptome durchgehen und protokollieren können. Anschließend sollen sie täglich nach dem Aufstehen und gegebenenfalls vor Einnahme der Medikamente eine Schätzung ihres Peak-Flow-Wertes abgeben, und danach den tatsächlichen Peak-Flow-Wert ermitteln. In regelmäßigen Gruppentreffen werden die Ergebnisprotokolle durchgesprochen, gerade im Hinblick auf die Abhängigkeit der Schätzgüte von der körperlichen Befindlichkeit.

\section{Modul Selbstsicherheitstraining}

Dieses Modul baut auf den Stunden 7-8 („Krankheitsbewältigung“) des Intensivkurses auf und richtet sich an Patienten, die entweder ein krankheitsbedingtes Sozialvermeidungsverhalten zeigen oder ihr Selbstmanagement aus sozialen Ängsten nicht adäquat durchführen. In diesem Training können auch krankheitskonkordante sozial- und agoraphobische Haltungen thematisiert werden. Gegebenenfalls wird dieses Modul ergänzt durch eine psychotherapeutische Behandlung. Ziele dieses Moduls sind es, andere Menschen über die eigene Erkrankung informieren und nötiges Selbstmanagement in der Öffentlichkeit praktizieren zu können sowie die Verbesserung des Bedürfnisausdrucks und der Durchsetzungsfähigkeit gegenüber medizinischen Fachpersonen. Dies wird in Standardrollenspielen (Inhalieren am Arbeitsplatz; Umgang mit Rauchen in Gruppen etc.) sowie anliegenorientierten Rollenspielen erarbeitet und geübt. 


\section{Modul Selbstmanagement}

Dieses Modul ist für Patienten konzipiert, die einerseits aufgrund eines instabilen Asthmaverlaufes bzw. der Notwendigkeit einer langfristigen und z.T. umfangreichen Medikation einen erhöhten Selbstmanagementbedarf haben oder andererseits aufgrund mangelnder Selbstmanagementfähigkeiten bereits vermeidbare Notfallsituationen oder Krankenhausaufenthalte durchlaufen haben. Die Ursache dieser mangelnden Selbstmanagementfähigkeiten kann sowohl in einem einfachen Wissen- und Fertigkeitsdefizit liegen, andererseits aber auch durch psychologische Hemmnisse bedingt sein (Krankheitsverdrängung, Folgen einer inadäquaten Laientheorie). Zur Verbesserung des Selbstmanagements erfolgt eine systematische Vertiefung der Themen „Selbstkontrolle“ und „ärztlich begleitetes Selbstmanagement“, welche bereits im Wochenkurses „Asthma bronchiale“ erörtert wurden. In dieser wöchentlich stattfindenden Gruppe können alle positiven oder negativen Erfahrungen mit der eigenständigen Therapieanpassung zur Sprache kommen. Insbesondere sollen auch Hemmungen und Widerstände thematisiert werden können. In dieser Gruppe sollen verstärkt Kompetenz- oder Fertigkeitsdefizite mit übenden (z.B. erfolgsbetontes Trainieren von Selbstmanagement und Notfalltechniken) und verhaltenstherapeutischen Verfahren (z. B. Verstärkung, Modelllernen, Erarbeiten von Verhaltensketten, Hilfen zum Alltagstransfer, Merkhilfen) aufgebaut werden. Die Einstellungen des Patienten zu seiner Erkrankung, deren Behandlung und seinen Bewältigungsmöglichkeiten (Krankheitsakzeptanz, Kontrollüberzeugungen, Selbstwirksamkeit) lassen sich mit kognitiven Interventionsverfahren wirksam beeinflussen.

\section{Modul: Gruppe „Nichtraucher werden“}

Obwohl Nichtraucher-Trainingsprogramme bisher eher unbefriedigende Ergebnisse zeigen, sind sie ein wichtiger Bestandteil eines umfassenden Verhaltenstrainingsprogrammes. Diese Gruppe ist konzipiert als eine Art Selbsthilfetraining mit Moderation. Ganz bewusst wird nicht von einem „Training“ gesprochen, da dies für die Patienten gelegentlich assoziiert ist mit einem Programm, das lediglich absolviert werden muss, um Nichtraucher zu werden.

Die erste Stunde dient dem Einstieg in die Gruppe; die weiteren Stunden finden einmal wöchentlich für die Dauer des Aufenthalts statt. Zu Beginn werden Einstellungsaspekte besprochen und die Bereitschaft, mit dem Rauchen aufzuhören, eruiert und transparent gemacht. Ergebnis dieses Prozesses ist eine Entscheidung, ob und wenn ja, wie mit dem Rauchen aufgehört werden soll. Diese wird in der zweiten Stunde besprochen. Prinzipiell werden die Reduktionsmethode und die Schlußpunktmethode (mit und ohne NikotinSubstitution) mit den Patienten erarbeitet. In den folgenden Stunden unterstützen sich die Patienten wechselseitig in ihrer Entscheidung und der praktischen Durchführung, das Rauchen deutlich einzuschränken oder besser ganz einzustellen. Großer Wert wird auf die verhaltenstherapeutische Erarbeitung von Gewohnheitsänderungen gelegt sowie den Aufbau von Genussfähigkeit.
Körperliche Trainingstherapie als praktisches Übungsfeld des Patientenverhaltenstrainings

Körperliches Training ist fester Bestandteil aller stationären pneumologischen Rehabilitationsprogramme. Durch die systematische Integration der speziell für Atemwegskranke konzipierten körperlichen Trainingstherapie in das Patientenverhaltenstrainingskonzept erschließt sich ein wichtiges praktisches psychosoziales Übungsfeld, da hier wesentliche Aspekte alltagsnah und unter kontrollierter Konfrontation mit dem Leitsymptom Belastungsintoleranz in der Gruppe eingeübt und optimiert werden. So kann ein adäquates Verhalten in Belastungssituationen erprobt werden, wobei die grundlegende Fertigkeiten „Selbstkontrolle“ (Peak-Flow-Meter, Borg-Skala, Interozeption u.a.) und der richtige Gebrauch der inhalativen Medikamente (z.B. Anwendungszeitpunkt vor dem Training, Applikationstechnik, Anwendung in der Öffentlichkeit) nochmals konkret geübt wird. Die körperliche Trainingstherapie ist daher ein ganz wesentliches praktisches Übungsfeld des Patientenverhaltenstrainings.

\section{Zusammenfassung und Ausblick}

Nach annähernd 15-jähriger Erfahrung betrachten wir Patientenschulung bei obstruktiven Atemwegserkrankungen mehr denn je als unverzichtbaren Basisbestandteil eines sinnvollen Therapiekonzeptes. Solche Programme müssen jedoch - gerade im Rahmen einer mehrwöchigen stationären Rehabilitationsbehandlung - individuell den Bedingungen und Bedürfnissen der Patienten angepasst werden. Notwendig erschien uns daher das Erarbeiten einer breiten Palette von unterschiedlichen - für jeden Patienten individuell miteinander kombinierbaren Schulungs- und Verhaltenstrainingsmodulen, die sowohl Wissen als auch praktische Fertigkeiten vermitteln und einüben und mit deren Hilfe auch Selbstmanagementfähigkeiten wie psychosoziale Kompetenzen erworben werden können.

- Diese Module müssen strukturiert, standardisiert, pädagogisch ausgefeilt und in ihrer Wirksamkeit evaluiert sein.

- Für jedes Modul sollte ein Kursheft für die Patienten ausgegeben werden.

- Eine Lernzielkontrolle sollte Schulungsbestandteil sein, um ggf. vorhandene Defizite gezielt angehen zu können.

Selbstverständlich erfordert dieses System einen vermehrten Personal- und Sachaufwand, der in dieser Form nur in größeren Reha-Kliniken zu leisten ist. Gerade diese RehaKliniken würden aber ihrer speziellen Aufgabe einer optimalen rehabilitativen Gesamtversorgung nicht gerecht, wenn sie sich bezüglich einer ihrer Kernaufgaben (Patientenverhaltenstraining) an den hier deutlich beschränkteren zeitlichen, räumlichen und personellen Ressourcen der Akutkrankenhäuser bzw. den ambulanten Schulungsmöglichkeiten orientierten. 


\section{Literatur}

${ }^{1}$ Petermann F. Patientenschulung und Patientenberatung - Ziele, Grundlagen und Perspektiven. In: Petermann F (Hrsg) Patientenschulung und Patientenberatung: ein Lehrbuch (2. Aufl). Göttingen: Hogrefe, 1997: 3-21

${ }^{2}$ Wettengel R, Berdel D, Hofmann D, Krause J, Kroegel C, Kroidl RF, Leopold W, Lindemann H, Magnussen H, Meister R, Morr H, Nolte D, Rabe KF, Reinhardt D, Sauer R, Schultze-Werninghaus G, Ukena D, Worth H. Asthmatherapie bei Kindern und Erwachsenen; Empfehlungen der Deutschen Atemwegsliga in der Deutschen Gesellschaft für Pneumologie. Medizinische Klinik 1998; 93: $639-650$

${ }^{3}$ National Heart, Lung and Blood Institute, National Institutes of Health. International consensus report on diagnosis and management of asthma. European Respiratory Journal 1992; 5: 601 641

${ }^{4}$ British Thoracic Society, Research Unit of Royal College of Physicians of London, King's Fund Centre. National Asthma Campaign: Guideline for management of asthma. Thorax 1994; 48: S1 - S24

${ }^{5}$ Sheffer AL (ed). Global strategy for asthma management and prevention (NHLBI/WHO Workshop Report, National Heart, Lung and Blood Institute). Bethesda: NHLBI-Publication, 1995: Number $95-3659$

${ }^{6}$ National Institutes of Health, National Heart, Lung and Blood Institute. Clinical Practice Guidelines, Expert Panel Report 2: Guidelines for the Diagnosis and Management of Asthma. Bethesda: NIH-Publication, 1997: Number 97-4051

${ }^{7}$ Ferguson GT, Cherniak RM. Management of chronic obstructive pulmonary disease. New England Journal of Medicine 1993; 328: $1017-1022$

${ }^{8}$ Siafakas NM, Vermeire P, Pride NB, Paoletti P, Gibson J, Howard P, Yernault JC, Decramer M, Higenbottam T, Postma DS et al.. (The European Respiratory Society Task Force): Optimal assessment and management of chronic obstructive pulmonary disease (COPD). Eur Respir J 1995; 8: 1398 - 1420

${ }^{9}$ ATS Statement. Standards for the diagnosis and care of patients with chronic obstructive pulmonary disease. Am J Respir Crit Care Med 1995; 152: S77-S120

${ }^{10}$ Fireman P, Friday GA, Gira C, Vierthaler WA, Michaels L. Teaching self-management skills to asthmatic children and their parents in an ambulant care setting. Pediatrics 1981; 68: $341-348$

${ }^{11}$ Lewis CE, Rachelefsky G, Lewis MA, de la Sota A, Kaplan M. A randomized trial of asthma care training for kids. Pediatrics 1984; 74: $478-486$

${ }^{12}$ Clark NM, Feldman $\mathrm{CH}$, Evans D, Levison MJ, Wasilewski Y, Mellins RB. The impact of health education on frequency and cost of health care use by low income children with asthma. J Allergy Clin Immunol 1986; 78: 108-115

${ }^{13}$ Mühlhauser I, Richter B, Kraut D, Weske G, Worth H, Berger M. Evaluation of a structured treatment and teaching programme on asthma. Journal of Internal Medicine 1991; 230: 157-164

${ }^{14}$ Mayo RH, Richmann J, Harris HW. Result of a program to reduce admissions for adult asthma. Ann Intern Med 1990; 112: $864-$ 871

${ }^{15}$ Wilson SR, Scamagas P, German DF, Hughes GW, Lulla S, Coss S, Chardon L, Thomas RG, Starr-Schneidkraut N, Stancavage FB. A controlled trial of two forms of self-management education for adults with asthma. The American Journal of Medicine 1993; 94 : $564-576$

${ }^{16}$ Ignacio-Garcia JM, Gonzales-Santos P. Asthma self-management education program by home monitoring of peak expiratory flow. Am J Respir Crit Care Med 1995; 151: 353 - 359

${ }^{17}$ Lahdensuo A, Haahtela T, Herrala J, Kava T, Kiviranta K, Kuusisto P, Peramaki E, Poussa T, Saarelainen S, Svahn T. Self management of asthma with peak flow meter vs. traditional treatment. BMJ 1996; 312: $748-752$

${ }^{18}$ Laukandt I, Kaspar P, Petro W. Patiententraining in der pneumologischen Rehabilitation verbessert die Lebensqualität bei Patienten mit obstruktiven Atemwegserkrankungen. Pneumologie 1996; 50: 122

${ }^{19}$ Ringsberg KC, Wiklund I, Wilhelmsen L. Education of adult patients at an "asthma school': effects on quality of life, knowledge and need for nursing. Eur Respir J 1990; 3: 33 - 37

${ }^{20}$ Trautner C, Richter B, Berger M. Cost-effectiveness of a structured treatment and teaching programme on asthma. Eur Respir J 1993; 6: $1485-1491$

${ }^{21}$ Volmer T. Wirtschaftlichkeitsüberlegungen bei Patientenschulungen. In: Petermann F (Hrsg) Patientenschulung und Patientenberatung: ein Lehrbuch (2. Aufl.). Göttingen: Hogrefe, 1997: $101-120$

${ }^{22}$ Devine EC. Meta-Analysis of the Effects of Psychoeducational Care in Adults with Asthma. Research in Nursing \& Health 1996; 19: $367-376$

${ }^{23}$ Watson PB, Town GI, Holbrook N, Dawn C, Toop LJ, Drennan CJ. Evaluation of a self-management plan for chronic obstructive pulmonary disease. Eur Respir J 1997; 10: 1267-1271

${ }^{24}$ Devine EC, Pearcy J. Meta-analysis of the effects of psychoeducational care in adults with chronic obstructive pulmonary disease. Patient Education and Counseling 1996; 29: 167- 178

${ }^{25}$ Arbeitsgruppe Patientenschulung der Deutschen Gesellschaft für Pneumologie und Deutsche Atemwegsliga in der Deutschen Gesellschaft für Pneumologie (Federf.: Pe. Empfehlungen zum strukturierten Patiententraining bei obstruktiven Atemwegserkrankungen. Medizinische Klinik 1995; 90: 515-519

${ }^{26}$ Hilton S, Sibbald B, Anderson HR, Freeling P. Controled evaluation of the effects of patient education on asthma morbidity in general practice. The Lancet 1986; i: $26-29$

${ }^{27}$ Müller C, Schwiersch M, Schultz K. Sport- und Verhaltenstherapie bei Asthma und chronisch-obstruktiver Bronchitis. Pneumologie 1999; 53: 229-231

${ }^{28}$ Worth H. Patientenschulung mit asthmakranken Erwachsenen. In: Petermann $\mathrm{F}$ (Hrsg). Patientenschulung und Patientenberatung: ein Lehrbuch (2. Aufl.). Göttingen: Hogrefe, 1997: 143-155

${ }^{29}$ Münks-Lederer C, Dhein Y, Richter B, Worth H. Langzeiteffekt eines strukturierten, ambulanten Asthma-Behandlungs- und Schulungsprogramms (AFAS). Pneumologie 1997; 51: 217

${ }^{30}$ Münks-Lederer C, Dhein Y, Richter B, Worth H. Evaluation eines strukturierten, ambulanten Asthma-Behandlungs- und Schulungsprogramm (AFAS). In: Petro, W Patientenverhaltenstraining. München-Deisenhofen: Dustri, 1997: 65 - 74

${ }^{31}$ Dhein Y, Scharcher C, Münks-Lederer C, Birkenmeyer A, Worth $\mathrm{H}$. Evaluation eines ambulanten und strukturierten AsthmaSchulungsprogramms (AFAS). Pneumologie 1999; 53: S21

${ }^{32}$ Dhein Y, Münks-Lederer C, Worth H. Ambulantes Fürther Schulungsprogramm für Patienten mit chronisch obstruktiver Bronchitis und Lungenemphysem (AFBE). In: Petro, W (Hrsg.) Patientenverhaltenstraining. München: Dustri, 1997: 56-64

33 Prittwitz M, Betz HP, Lauber B, Härtl W, Selke J, Kaspar P, Morawa R, Fleischer M, Petro W. Bad Reichenhaller Modell der Patientenschulung Asthma, Bronchitis, Emphysem. In: Petro, W (Hrsg.) Patientenschulung für Atemwegserkrankte. München: Dustri, 1989

${ }^{34}$ Petro W, Holländer P, Betz HP, Haman B, Lauber B, Mzyk C, Prittwitz M. Patientenschulung in der pneumologischen Rehabilitation steigert den therapeutischen Erfolg. Atemw.-Lungenkrankh 1995; 21: 49-58

${ }^{35}$ Schultz K, Stark HJ, Petro W. Modulares, maßgeschneidertes Patientenverhaltenstraining. In: Petro, W (Hrsg.) Patientenverhaltenstraining bei obstruktiven Atemwegserkrankungen. München: Dustri, 1997: 142-162 
${ }^{36}$ Gallefoss F, Bakke PS, Rsgaard PK. Quality of life assessment after patient education in a randomized controlled study on asthma and chronic obstructive pulmonary disease. Am J Respir Crit Care Med 1999; 159: $812-817$

${ }^{37}$ Petermann F, Mühlig S. Grundlagen und Möglichkeiten der Compliance-Verbesserung. In: Petermann F (Hrsg.) Compliance und Selbstmanagement. Göttingen: Hogrefe, 1998: 73-102

${ }^{38}$ Mühlig S, Schultz K, de Vries U, Petermann F. Grundlagen der Patientenschulung bei Asthma. In: Petermann F \&Waschburger P (Hrsg.) Asthma bronchiale. Göttingen: Hogrefe, 2000

${ }^{39}$ Schultz K, Petro W. Patientenverhaltenstraining bei obstruktiven Atemwegserkrankungen Erwachsener. In: Petro W: Pneumologische Prävention und Rehabiliation, 2. Auflage. Springer Verlag: Heidelberg, 2000

${ }^{40}$ Stark HJ, Schultz K, Petro W. Eine lernzielorientierte Peak-FlowMeter-Schulung führt zu einem signifikanten Wissenszuwachs bei Patienten mit COPD. Pneumologie 1996; 50: S 158

${ }^{41}$ Stark HJ, Schultz K, Petro W. The inter-trainer-variability in the context of pneumological patients education is minimized by a standardized training program based on special learning objectives. Am J Respir Crit Care Med 1996; 153: A 85

42 Roider E, Stark HJ, Schultz K, Petro W. Neue Aufgaben des Pflegepersonals bei der Schulung von Patienten mit chronisch obstruktiven Atemwegserkrankungen. Pneumologie 1996; 50: S158

${ }^{43}$ Schultz K, Petro W. Der spezielle Wissens- und Fertigkeitsstand von ambulanten Patienten mit Indikation zur Peak-Flowmetrie ist insuffizient, kann aber durch ein strukturiertes Patientenverhaltenstraining deutlich gebessert werden. Tagungsband des 8 . Rehabilitationswissenschaftlichen Kolloquiums des VDR. Norderney, 1999 Frankfurt, 1999: 334-336

${ }^{44}$ Stark HJ, Schultz K, Petro O, Petro W. Development of a Computer-Supported Multi-Media Peak Flow Meter Patient Training Program. Am J Respir Crit Care Med 1997; 155: A570

${ }^{45}$ Stark HJ, Schultz K, Petro W. Neue Wege in der Patientenschulung. In: Petro W Patientenverhaltenstraining. München: Dustri, 1997: $163-175$

${ }^{46}$ Mehlmann B, Stark HJ, Roider E, Schultz K, Petro W. Short-Term Evaluation of a Computer-Supported Multi-Peak Flow Meter Patient Training Program (BREATH). Eur Respir J 1997; 10: 460s

${ }^{47}$ Schultz K, Stark HJ, Petro W. Neue Schulungsaufgaben in der pneumologischen Rehabilitationsmedizin. Atmw.-Lungenkrankh 1996; $22: 38-44$

${ }^{48}$ Schultz K, Stark HJ, Petro W. Das Bad Reichenhaller Modell des Allergikertrainings, Tagungsband des Rehabilitationswissenschaftlichen Kolloquiums des VDR 1996. Frankfurt, 1996

${ }^{49}$ Schultz K, Stark HJ, Petro W. Standardisiertes Trainingsprogramm für Hausstaubmilbenallergiker. Pneumologie 1996; 50: S158

${ }^{50}$ Schultz K, Hacker A, Räder H, Petro W. 3 Jahre Erfahrung mit einem modular strukturierten Schulungsprogramm für Patienten mit allergischen Atemwegserkrankungen. Allergologie 1999; 22: $223-229$

${ }^{51}$ Schultz K, Stark HJ, Petro W. Development of a standardized training program for patients with an indication for long-term oxygen therapy (LTOT). Am J Respir Crit Care Med 1996; 153: A 783

${ }^{52}$ Schultz K, Kühler U, Benteler J, Petro W. Three Years of Experience with a Standardized Education Program for Patients with an Indication for Long-Term Oxygen Therapy (LTOT). In: Tagungsband des ECRR. Berlin, 1998 Frankfurt, 1998

${ }^{53}$ Benteler J, Schultz K, Stark HJ, Petro W. Two Years Experience with a Standardized Training Program for patients with an Indication for Long-Term Oxygen Therapy (LTOT). Eur Respir J 1997; 10: 460s
${ }^{54}$ Vergeret J, Tunon De Lara M, Douvier JJ, Freour P, Cardinaud JP, Courty G, Taytard A. Compliance of COPD patients with long term oxygen therapy. Eur J Respir Dis Suppl 1986; 146: 421 - 425

${ }^{55}$ Howard P, Waterhouse JC, Billings CG. Compliance with longterm oxygen therapy by concentrator. Eur Respir J 1992; 5: 128 129

${ }^{56}$ Walshaw MJ, Lim R, Evans CC, Hind CR. Factors influencing the compliance of patients using oxygen concentrators for longterm home oxygen therapy. Respir Med 1990; 84: 331 - 333

${ }^{57}$ Frey JG, Kaelin RM, De Werra M, Jordan B, Tschopp JM. Continuous oxygen therapy at home. Observations of oxygen users after an instruction program. Rev Mal Respir 1992; 9: $301-305$

58 Pepin JL, Barjhoux CE, Deschaux C, Brambilla C. ANTADIR Working Group on Oxygen Therapy. Long-term oxygen therapy at home. Compliance with medical prescription and effective use of therapy. Chest 1996; 109: 1144-1150

59 Petermann F. Asthma bronchiale. Göttingen: Hogrefe, 1999

${ }^{60}$ Dahme B, König R, Nußbaum B, Richter R. Haben Asthmatiker Defizite in der Symptomwahrnehmung? Quasi-experimentelle und experimentelle Befunde zur Interozeption der Atemwegsobstruktion. Psychotherapie, Psychosomatik und Medizinische Psychologie 1991; 41: 490-499

${ }^{61}$ Dahme B, Richter R, Mass R. Interoception of respiratory resistance in asthmatic patients. Biological Psychology 1996; 42: $215-229$

62 Vogt M, Schandry R. Symptomwahrnehmung bei Asthmapatienten. Pneumologie 1995; 49: 316-321

${ }^{63}$ Rietveld S. Symptom Perception in Asthma: A Multidisciplinary Review. In: Journal of Asthma 1998; 35: 137-146

\section{Dr. med. K. Schultz}

Fachklinik Allgäu

Verhaltensmedizinisches Zentrum für Pneumologie,

Allergologie, Dermatologie und Psychosomatik

Peter-Heel-Str. 29

87459 Pfronten

E-mail: kschultz@ahg.de 